\title{
Correlação entre fissura labiopalatina e processamento auditivo (central)*
}

\section{Correlation between cleft lip and palate and (central) auditory processing}

Yandra Mesquita Pereira Cardoso ${ }^{1}$ Maria Cristina de Almeida Freitas Cardoso ${ }^{2}$ Maria Inês Dornelles da Costa-Ferreira ${ }^{3}$

* Recebido em: $12 / 02 / 14$

Aprovado em: 07/11/14

1 Bacharel em Fonoaudiologia pelo Centro Universitário Metodista do Instituto Porto Alegre - IPA, Porto Alegre, RS, Brasil;

2 Fonoaudióloga Clínica; Doutora em Gerontologia Biomédica pela Pontifícia Universidade Católica do Rio Grande do Sul - PUCRS, Porto Alegre, RS, Brasil; Professora do curso de Fonoaudiologia e do Programa de Pós Graduação - Ciências da Reabilitação da Universidade Federal de Ciências da Saúde de Porto Alegre UFCSPA, Porto Alegre, RS, Brasil;

3 Fonoaudióloga Clínica; Doutora em Linguística Aplicada pela Pontifícia Universidade Católica do Rio Grande do Sul - PUCRS, Porto Alegre, RS, Brasil; Docente do curso de Fonoaudiologia do Centro Universitário Metodista do Instituto Porto Alegre - IPA, Porto Alegre, RS; Docente do curso de Fonoaudiologia da Faculdade Nossa Senhora de Fátima, Caxias do Sul, RS, Brasil.

\section{Resumo}

As fissuras labiopalatinas são malformações congênitas que comprometem os aspectos funcionais orofaciais, bucais, articulatórios e estéticos, podendo ocorrer problemas auditivos por otites médias de repetição e disfunções da tuba auditiva, afetando o processamento auditivo. $\mathrm{O}$ objetivo deste estudo é averiguar na literatura a ocorrência de transtorno de processamento auditivo em portadores de fissuras labiopalatinas. Trata-se de uma revisão crítica da literatura nas bases de dados: Scielo; Bireme e ScienceDirect. Os descritores utilizados foram: fissura labiopalatinas, transtornos do processamento auditivo e audibilidade. Os artigos analisados apontam a possibilidade de ocorrência de transtorno de processamento auditivo devido às malformações associadas às fissuras labiopalatinas e direcionam a reabilitação para a protetização e o treino de habilidades auditivas. Os portadores desta malformação apresentam um comprometimento nas estruturas anatomofisiológicas e de audibilidade que podem desencadear transtorno de processamento auditivo.

Palavras-chave: Audição. Perda auditiva central. Fenda labial. Fissura palatina. Audibilidade.

\begin{abstract}
The clefts lip and palate are congenital malformations, which compromise the oral facials' functional aspects, mouths aspects, articulatory and aesthetics' which may occur, including, hearing problems, recurrent otitis media by and dysfunction of the Eustachian tube which consequently can affect your auditory processing. The aim of this study is a literature investigating of the occurrence of auditory processing disorder in clefts lip and palate. This is a critical review of literature of the data's bases: Scielo; Bireme; Direct Scients. The descriptors used were: cleft lip and palate, auditory processing disorders and audibility. Articles analyzed pointed out the possibility of auditory processing disorder through malformations associated with the clefts lip and palate and direct the rehabilitation for prostheses and training of the auditory skills. Clefts lip and palate holders feature a commitment in audibility and anatomy and physiological structures can trigger auditory processing disorders.
\end{abstract}

Keywords: Hearing. Hearing Loss. Central. Cleft Lip. Cleft Palate. 


\section{Introdução}

As fissuras labiopalatinas (FLP) são malformações congênitas que acometem os indivíduos durante o seu desenvolvimento embrionário entre a $9^{\mathrm{a}}$ e $12^{\mathrm{a}}$ semana gestacional. Tal malformação compromete os seus portadores quanto aos aspectos funcionais orofaciais, bucais, articulatórios e estéticos, podendo ocorrer, entre eles, problemas auditivos, por otites médias de repetição e disfunções da tuba auditiva que consequentemente podem afetar o processamento auditivo (PA) (AMARAL; MARTINS; SANTOS, 2010).

De acordo com os dados do Sistema de Informações sobre Nascidos Vivos, em 2011 foram notificados 1593 nascimentos de crianças com fenda labial e fissura palatina no Brasil (BRASIL, 2011). As FLP acometem cerca de 2,1 nascidos vivos entre os asiáticos, 1 para cada 1000 nascidos vivos entre os indivíduos brancos, e de 0,41 casos por 1000 nascidos vivos entre os negros (TEWFIK, 2011).

Neves, Monteiro e Giwa (2002), ao realizarem uma pesquisa na cidade de São José dos Campos (SP), encontraram a existência de um maior número de indivíduos com fissura transforame incisivo que a pré e pós-forame incisivo, de um número maior de pessoas do sexo masculino portadores de FLP e da não existência de antecedentes familiares de fissura. Tais indicadores de prevalência são referenciados, ainda, com a ocorrência da morbidade de distúrbios articulatórios compensatórios, como descritos por Pamplona et al. (2000) cuja frequência mostrou-se significativamente alta para o atraso de desenvolvimento de linguagem.

No decorrer do desenvolvimento neuropsicológico, a criança interage com meio ambiente e estabelece a comunicação por meio da linguagem. Essa interação favorece a comunicação verbal oral a partir da integridade auditiva e dos aspectos neuropsicológicos.

A audibilidade está presente desde os sons ambientais, que aparecem em diferentes intensidades desde as mais baixas, quase inaudíveis até as mais altas, que podem provocar desconforto. Além disso, a audição é um sentido essencial à comunicação (LUZ; COSTA-FERREIRA, 2011) e, por isso, na fase da aquisição da linguagem admite-se um padrão de normalidade de $15 \mathrm{dBNA}$, visando à percepção dos traços acústicos mínimos (NORTHERN; DOWNS, 2005).

O PA é caracterizado por um conjunto de habilidades específicas que o indivíduo necessita para interpre- tar o que ouve, sendo mediadas pelos centros auditivos localizados no tronco encefálico e cérebro (ASHA, 2005; AAA, 2010).

O desenvolvimento do PA se inicia pelo contato da criança com os vários tipos de sons do ambiente e, quando esse processo não ocorre, por uma dificuldade de processamento perceptual da informação no Sistema Nervoso Central (SNC), tem-se um Transtorno de Processamento Auditivo (TPA), (ACA, 2012)

As habilidades alteradas no TPA podem ser classificadas em: localização auditiva, habilidade avaliada por meio de testes de interação binaural; fechamento auditivo, habilidade avaliada pelos testes monoaurais de baixa redundância; análise e síntese auditivas, avaliadas pelos testes dicóticos; e processamento auditivo temporal, habilidade avaliada por maior de testes de sequencialização e resolução temporal (ASHA, 2005; VARGAS et al, 2014).

Visto que as malformações labiopalatinas podem acometer as estruturas que compõem o trato auditivo, esta revisão crítica da literatura tem como objetivo averiguar o TPA em portadores de FLP.

\section{Método}

Este artigo é uma revisão de literatura por narrativa, cujos critérios de inclusão foram os descritores de FLP e TPA associados, publicados nos últimos 10 anos, nos idiomas inglês e português. Foram excluídos artigos que definiam exclusivamente as FLP ou a perda auditiva central. A busca dos artigos foi realizada nas bases Scielo, Bireme e Science Direct. Os descritores utilizados foram: audição, perda auditiva central, fissura palatina e fenda labial, com o intuito de identificar artigos que relacionassem as FLP aos TPA. Foi necessário o uso de obras clássicas publicadas sobre o assunto para complementar esse estudo.

\section{Transtornos de audição nas FLP}

Grande parte dos indivíduos portadores de FLP apresenta alguma alteração auditiva, que vem a ser confirmada com os exames de audiometria tonal e vocal e medidas de imitância acústica (SANTOS; PIAZENTIN-PENNA; BRANDÃO, 2011; CEROM et al, 2013; MAN$\mathrm{ZI}$ et al, 2013). Frequentemente, encontram-se ligadas aos transtornos da tuba auditiva em indivíduos com FLP. A tuba auditiva (TA) é um canal que faz ligação entre a ca- 
vidade timpânica e a nasofaringe, medindo entre 35 a 38 milímetros de comprimento, é formada por uma camada de tecido ósseo (um terço do seu comprimento) e de tecido fibrocartilaginoso (dois terços). A porção óssea da TA é revestida por uma mucosa com epitélio colunar $80 \%$ ciliada, com células caliciformes secretoras. A sua porção cartilaginosa é revestida por mucosa com epitélio colunar pseudoestratificado ciliado, epitélio cuboide ciliado e células caliciformes com "glândulas tubuloacinosas que secretam muco na luz da tuba". No recém-nascido, a cartilagem é a hialina e no adulto a elástica (ZORZETTO, 2006).

A função da TA é manter o equilíbrio das pressões de ar entre as orelhas externa e média, permitindo a ventilação dos espaços pneumatizados do osso temporal e proteger contra possíveis agressões bacterianas (RUSSO; SANTOS, 2005; ZORZETTO, 2006). Assim, ela se mantém basicamente fechada, evitando o refluxo de secreções e bactérias da nasofaringe para a orelha média, além de proteger flutuações de pressões durante a deglutição, tosse e respiração. O processo de abertura da TA necessita de uma contração do músculo tensor do véu-palatino, e uma contração conjunta entre os músculos tensores e elevador do véu-palatino (PROCTOR, 1973; CASSELBRANT et al, 1988).

Em uma pesquisa de análise de prontuários de aproximadamente 150 sujeitos portadores de FLP, Santos, Piazentin-Penna e Brandão (2011) selecionaram aleatoriamente 119 (79\%) do gênero masculino e 31 (21\%) do gênero feminino, com idade igual ou superior a 4 anos, com fissura labiopalatina operada e com indicação de cirurgia otológica. Com esses dados, os autores dividiram os sujeitos nos seguintes grupos: grupo I: composto de 50 sujeitos submetidos à avaliação audiológica e microcirurgia otológica com inserção de tubo de ventilação; grupo II: composto de 50 sujeitos submetidos à avaliação audiológica e realização da timpanoplastia; grupo III: composto de 50 sujeitos submetidos à avaliação audiológica e realização da timpanomastoidectomia. Por meio da audiometria tonal limiar, os achados desses autores foram classificados quanto ao tipo e grau da perda auditiva. Os autores verificaram que $83 \%(n=125)$ apresentaram algum tipo de queixa (atual e/ou pregressa) e que, no grupo II, houve maior ocorrência de perda auditiva condutiva unilateral (48\%), seguida de perda auditiva condutiva bilateral (34\%) e perda auditiva mista bilateral (6\%). O estudo permitiu aos autores concluir que a maioria das alterações foi compatível com problemas de orelha média, com perda auditiva do tipo condutiva, de grau leve e bilateral, independentemente da indicação cirúrgica.
O estudo de Amaral, Martins e Santos (2010), que avaliou 44 crianças de ambos os sexos, entre 8 e 14 anos de idade, portadoras de FLP, constatou que a perda auditiva com maior incidência é a do tipo condutiva, afetando $13,6 \%$ das crianças pesquisadas, correspondendo com alguma alteração de orelha média. Esses dados foram confirmados por meio de um questionário respondido pelos pais das crianças, no qual há o relato de que os filhos tiveram, no decorrer dos anos, vários episódios de otites médias agudas.

Carvalhal (2003), ao descrever as alterações otológicas de 180 sujeitos portadores de fissura labiopalatina ou palatina isolada, detectou $130(72,2 \%)$ desses sujeitos sem queixas otorrinolaringológicas na primeira avaliação. Por meio da otoscopia realizada nessa pesquisa, o autor detectou otite média com efusão em 80 sujeitos (46,2\%); otite média crônica colesteatomatosa em 11 (6,4\%); otite média não-colesteatomatosa em 9 (5,2\%); e retração da membrana timpânica de grau moderado/severo em 24 sujeitos (13,9\%). Os seus resultados mostram uma relação entre a idade e a presença de alterações auditivas, pois, com o passar dos anos, há uma diminuição na ocorrência da efusão de orelha média, mas ocorre um aumento nas patologias mais crônicas como a retração da membrana timpânica moderado/severo e de otite crônica com ou sem colesteatoma. Devido a esses fatores, o autor afirma que a avaliação otorrinolaringológica deve ser realizada com frequência em pacientes com FLP para uma melhor intervenção e manutenção da orelha média.

Outros estudos também mostraram que grande parte dos pacientes com fissura palatina apresentava disfunção tubária, bem como verificaram a associação entre a queixa e a avaliação audiológica convencional (CEROM et al, 2013; MANZI et al, 2013).

\section{0 processamento auditivo nas FLP}

As habilidades do processamento auditivo dependem de capacidades inatas, e da relação do indivíduo com as experiências auditivas. Quando tais habilidades encontram-se alteradas, a criança provavelmente apresentará déficits de linguagem e até mesmo, dificuldades de aprendizado escolar. Os casos de perda auditiva condutiva, em crianças pequenas, podem provocar transtornos do processamento, gerando dificuldades de atenção e aprendizado da comunicação (LUZ; COSTA-FERREIRA, 2011).

Os TPA em crianças com FLP estão diretamente 
ligados aos eventos de otites médias de repetição, pois estas irão provocar perda auditiva condutiva, que consequentemente irão prejudicar as habilidades do PA (SHINN, 2003; LUZ; COSTA-FERREIRA, 2011).

Na pesquisa de Amaral, Martins e Santos (2010), que avaliou algumas habilidades de processamento auditivo em crianças com FLP, constatou-se que $72,7 \%$ das crianças exibiram alguma alteração, sendo que 45,5\% continham comprometimento no teste Dicótico de Dígitos. Outro estudo mostrou que tais crianças possuem baixo desempenho em testes de fusão auditiva quando comparadas com crianças que não possuem FLP (CASSAB; ZORZETTO, 2006).

As habilidades de localização sonora e discriminação estão relacionadas com a função de interação binaural, ou seja, a comunicação entre as duas orelhas. Nos casos de FLP, essa capacidade, possivelmente, será alterada por uma dificuldade auditiva periférica, assim sendo, por perda auditiva decorrente de otites como referido por Bellis (2003).

No estudo de Lemos et al. (2008), observou-se que as crianças com FLP, do gênero feminino, obtiveram desempenho inferior no teste Dicótico de Dígitos (etapa de escuta direcionada), quando comparadas às crianças do grupo controle, em ambas as orelhas.

Outras alterações do PA, em portadores de FLP, estão ligadas à resolução temporal, na qual é preciso detectar o intervalo de tempo entre os sons ou o menor tempo que o individuo precisa para discriminá-lo (SHINN, 2003). As alterações do PA relacionadas aos eventos de otites médias podem gerar dificuldades na identificação de pequenas variações acústicas da fala ou em entender uma mensagem, principalmente na época de aquisição da linguagem.

No estudo de Boscariol, André e Feniman (2009), que buscou avaliar as habilidades de PA em crianças com FLP, obteve-se resultados demonstrando que os piores desempenhos das crianças eram nos testes de Fusão Auditiva-Revisado (AFT-R), "Dicótico de Dígitos" (DD), "SSW" e no teste "PSI"/"SSI"/"MCI", e os melhores resultados foram nos testes de localização sonora, memória sequencial para sons não verbais e verbais e para "PSI"/"SSI"/"MCC".

Uma pesquisa verificou as condições de escuta de crianças com FLP em seis condições distintas e verificou que a condição no ruído torna-se mais difícil quando a atenção auditiva e a memória são requeridas (MANOEL et al, 2010).
Fenimam, Souza, Teixeira e Mondelli (2012) verificaram a percepção dos pais de crianças com FLP sobre a atenção auditiva de seus filhos e concluiu que a presença da patologia pode encontrar-se relacionada à atenção auditiva. Esse achado também foi confirmado por outro estudo em que as crianças com FLP apresentaram desempenho inferior no teste de atenção auditiva àquelas sem anomalia craniofacial apenas para a vigilância auditiva (LEMOS; FENIMAM, 2010). Do mesmo grupo, Moraes, Maximino e Fenimam (2011) verificaram a habilidade de atenção auditiva sustentada em crianças com FLP e transtorno fonológico. Das sete crianças com transtorno fonológico (41\%), duas (29\%) apresentaram alteração nos resultados do Teste da Habilidade de Atenção Auditiva Sustentada. Os autores concluíram que a habilidade de atenção auditiva sustentada nas crianças com fissura labiopalatina e transtorno fonológico não difere da habilidade de atenção auditiva sustentada de crianças com fissura labiopalatina sem transtorno fonológico.

Estudos eletrofisiológicos foram realizados com a mesma população clínica. Um deles investigou o potencial evocado auditivo de tronco encefálico e verificou latências absolutas da onda I normais em todos os pacientes, aumento das latências absolutas da onda III e V em dois e seis pacientes respectivamente; latências interpicos I-III, III-V e I-V aumentadas em quatro, três e oito pacientes, respectivamente (CASSAB et al, 2011). Por outro lado, uma pesquisa utilizou o mesmo potencial evocado auditivo e não encontrou alterações na via auditiva até o tronco encefálico, sugerindo a necessidade de avaliar níveis mais altos no sistema auditivo (ANTONELI et al, 2011)

\section{Discussão}

A incidência e a prevalência das FLP, assim como as comorbidades relacionadas são indicativos importantes para o acompanhamento do desenvolvimento da comunicação dos seus portadores.

Os portadores de FLP apresentam características anatomofuncionais que comprometem a integridade das vias auditivas, evidenciadas na configuração da TA, que apresenta uma falha no seu mecanismo de abertura, por uma falta de união das fibras musculares dos músculos tensor e elevador do véu-palatino (CASSELBRANT et al, 1998; CASSAB; ZORZETTO, 2006). Tal falha também compromete a funcionalidade do Esfíncter Velofaríngeo (EVF).

O músculo tensor do véu palatino é responsável 
pela abertura da TA, fazendo com que ocorra um movimento de abaixamento da porção anterior do véu, durante o processo de deglutição. Devido a isso, a integridade do palato e das estruturas que o compõem deve estar perfeita para que esse mecanismo ocorra com sucesso.

O EVF possui uma relação importante com a audição, pois se trata de uma cinta muscular que se localiza entre a orofaringe e a nasofaringe, compreendendo a musculatura do palato e das paredes lateral e posterior da faringe, possuindo uma ligação direta com a tuba auditiva (SILVA et al., 2008).

As alterações ou disfunção do EVF acontecem devido a uma insuficiência ou incompetência velofaríngea, ou seja, por uma malformação anatômica que ocasiona falta de tecido do palato mole ou por falta de competência neuromuscular no movimento das estruturas do EVF (TRINDADE; TRINDADE JUNIOR, 1996; JOHNS; ROHRICH; AWADA, 2003).

O mal fechamento do EFV e a falha na abertura da TA possibilitam a entrada de secreções da nasofaringe, dificultando a equalização das pressões entre orelhas externa e média, provocando otites de repetição, que podem ocasionar perda auditiva. Conforme Santos, Piazentin-Penna e Brandão (2011), para o funcionamento adequado da orelha média, é imprescindível que a TA "desempenhe corretamente as suas funções de drenagem, aeração e proteção da orelha média frente às secreções da nasofaringe".

A perda auditiva condutiva ocasionada pela orelha média com a presença de secreção por longo período de tempo gera algum grau de perda auditiva, provoca o abafamento das respostas de audibilidade e, consequentemente, dificulta a localização do som, pois o portador de FLP não consegue perceber auditivamente em que direção o som se encontra. Essa perda, em geral, é uma perda auditiva que se mostra com maior prejuízo nas frequências médias e graves $(250,500$ e $1000 \mathrm{~Hz})$ e tal dificuldade pode ser encontrada na produção dos fonemas fricativos que são relativamente agudos principalmente o /s/ e o /z/ (que se encontram entre as frequências de 4500 a 8000 $\mathrm{Hz}$, no português brasileiro). Esse dado foi confirmado por Cielo e Casarin (2008), que afirmam serem os mais afetados nas FLP assim como os fonemas /f/ e /v/ (COSTA-FERREIRA, 2015a).

De acordo com os pressupostos teóricos até agora apresentados, é evidente a preocupação com a integridade auditiva em pacientes com FLP, devido à ocorrência de perda auditiva condutiva, pois podem encontrar-se desatentos não atendendo ao chamado, ter dificuldades em acompanhar uma conversa com várias pessoas falando ao mesmo tempo ou até mesmo não conseguir escutar o professor numa sala de aula ruidosa, ter dificuldades para dar recados completos, apresentar substituições fonêmicas ou imprecisão articulatórias, realizar substituições grafêmicas e até mesmo, ter dificuldades de localização sonora.

O processamento auditivo é a eficiência e a efetividade com que o sistema nervoso central organiza a informação auditiva (ASHA, 2005; AAA, 2010). Tal eficiência ocorre da seguinte forma: a organização tonotópica da cóclea é a especificidade de frequência que ocorre por consequência das propriedades mecânicas da membrana basilar. Essa organização é repetida ao longo das vias auditivas do tronco encefálico até o córtex auditivo primário permitindo ao tronco encefálico salientar as características acústicas de frequência, intensidade e duração, necessárias à percepção da fala atrasando as demais como, por exemplo, o ruído de fundo. Além disso, o tronco encefálico compara as informações vindas de ambas as orelhas implicando a localização e a lateralização auditiva. O córtex auditivo primário, por sua vez, é especializado na codificação de eventos acústicos rápidos, sendo o local em que a discriminação das características acústicas mínimas dos sons da fala ocorre utilizando diferenças de intensidade e tempo de chegada do som (SHINN, 2007). Todo esse processo que é responsável pela discriminação auditiva pode ser avaliado pelos testes monoaurais de baixa redundância, testes de interação binaural e de processamento temporal (COSTA-FERREIRA, 2015a)

No estudo de Boscariol, André e Feniman (2009), os piores desempenhos estavam relacionados aos testes de Fusão Auditiva-Revisado (AFT-R), Dicótico de Dígitos (DD), SSW e no teste PSI ou SSI (MCI). Convém ressaltar que os testes DD e SSW não avaliam especificamente a discriminação auditiva, mas podem ser influenciados por ela, pois os testes dicóticos também envolvem áreas integrativas e de programação da resposta (COSTA-FERREIRA, 2015a).

Estudos abordados na sessão anterior verificaram prejuízo da atenção auditiva principalmente, a atenção sustentada (FENIMAM; SOUZA; TEIXEIRA; MONDELLI, 2012; LEMOS; FENIMAM, 2010; MORAES; MAXIMINO; FENIMAM, 2011). Convém ressaltar que todos os testes de processamento auditivo exigem algum nível de atenção sustentada para serem realizados.

Em relação ao tratamento as FLP, por provocar 
alterações nas estruturas do lábio e do palato, podem causar comprometimento auditivo, devido às otites médias recorrentes. A literatura referencia que o tratamento é controverso, encontrando-se (BOSCARIOL; ANDRÉ; FENIMAN, 2009; ZAMBONATO et al., 2011): o uso de antibióticos; inserção do tubo de ventilação; o acompanhamento otorrinolaringológico, audiológico e a reabilitação aural, com uso de aparelho de amplificação sonora individual (AASI). Em alguns casos, com perda auditiva neurossensorial associada, utilizaram o AASI, pois possuem história de otites médias e/ou tratamento por intervenção cirúrgica nos casos de otite médias recorrente. (ZAMBONATO et al., 2011).

Por fim, considera-se que o treinamento das habilidades auditivas envolvidas no PA mostra-se um importante aliado ao processo de reabilitação dos sujeitos portadores de FLP, pois, como ressaltam Lemos et al. (2008), as crianças com FLP apresentam períodos muito mais longos de privação sensorial, causados por otites médias, diferenciando-as daquelas sem a anomalia craniofacial. Dessa forma, a neurociência oferece suporte para a compreensão de um modelo de treinamento auditivo incorporando os processos bottom-up (melhora da recepção do sinal acústico) e top-down (estratégias linguísticas, cognitivas e metacognitivas) cujo objetivo é explorar a plasticidade auditiva e a reorganização cortical para maximizar a generalização e reduzir os déficits funcionais. O objetivo da terapia e as atividades a serem realizadas são baseados nos resultados da bateria comportamental de avaliação do processamento auditivo, na história individual e nos dados linguísticos e psicoeducacionais de cada paciente (COSTA-FERREIRA, 2015b). Além disso, pode envolver a abordagem direta (treinamento auditivo propriamente dito), estratégias comportamentais e modificações ambientais como o uso do Sistema de Frequência Modulada para melhorar a recepção do sinal acústico (ASHA, 2005; AAA, 2010).

\section{Considerações finais}

O processo de comunicação depende de fatores diversos e, dentre eles, as estruturas anatomofisiológicas, os aspectos neuropsicológicos e de audibilidade íntegros. Os portadores de FLP apresentam um comprometimento das estruturas anatomofisiológicas que desencadeiam comorbidades referentes, entre outras, a audibilidade. As habilidades alteradas no TPA, decorrentes da perda auditiva condutiva em pacientes com FLP, podem envolver várias funções, principalmente localização auditiva, o fechamento e figura-fundo, interação binaural e processamento auditivo temporal além de atenção auditiva sustentada diminuída. Concorda-se com os autores que mencionaram a necessidade de avaliar sistemas mais altos da via auditiva e com a proposição de uma reabilitação auditiva mais eficaz que envolva processos bottom- up e top-down, além de modificações ambientais. Outro aspecto a ser melhor investigado é o uso de tecnologia assistiva como o sistema de frequência modulada.

\section{Referências}

ACA (ACADÉMIE CANADIENNE D'AUDIOLOGIE). Canadian guidelines on auditory processing disorders in children and adults: assessment and intervention. 2012. Disponível em: <https://canadianaudiology.ca/ assets/docs/Canadian_Guidelines_on_Auditory_Processing_Disorder_in_Children_and_Adults_EN_2012_ new-site.pdf>. Acesso em: 30 out. 2014.

AMARAL, M. I. R.; MARTINS, J. E.; SANTOS, M. F. C. Estudo da audição em crianças com fissura labiopalatina não-sindrômica. Brazilian Journal of Otorhinolarynlogy, São Paulo, v. 76, n. 2, p. 164-171, mar./abr. 2010. Doi: $10.1590 / \mathrm{S} 1808-86942010000200004$

AAA (AMERICAN ACADEMY OF AUDIOLOGY). Guidelines for the Diagnosis, Treatment and Management of Children and Adults with Central Auditory Processing Disorder. 2010. Disponível em: <http://audiology-web.s3.amazonaws.com/migrated/CAPD\%20 Guidelines\%208-2010.pdf_539952af956c79.73897613. pdf $>$. Acesso em: 30 out. 2014.

ASHA (AMERICAN SPEECH-LANGUAGE-HEARING ASSOCIATION). Auditory Processing Disorders. Technical Report, 2005. [serial on the Internet]. Disponível em: <http://www.asha.org/members/deskref-journals/deskref/default>. Acesso em: 20 fev. 2014.

BALEN, S. Processamento auditivo central: aspectos temporais da audição e percepção acústica da fala. 1997. 176 f. Dissertação (Mestrado)-Pontifícia Universidade Católica de São Paulo, São Paulo, 1997.

BELLIS, T. J. Assessment and management of central auditory processing disorders in the educational setting: from science to practice. New York: Delmar Learning, 2003.

BOSCARIOL, M.; ANDRÉ, K. D.; FENIMAN, M. R. Crianças com fissura isolada de palato: desempenho nos testes de processamento auditivo. Brazilian Journal of Otorhinolaryngology, São Paulo, v. 75, n. 2, p. 213-220, mar./abr. 2009. 
BRASIL. Ministério da Saúde. DATASUS. Tabnet. 2011. Disponível em: <http://tabnet.datasus.gov.br/cgi/tabcgi. exe?sinasc/cnv/nvuf.def>. Acesso em: 20 fev. 2014.

CARVALHAL, L. H. S. K. Descrição das alterações otológicas de pacientes com fissura labiopalatina ou palatina isolada. 2003. 145 f. Dissertação (Mestrado)-Faculdade de Medicina do Rio Grande do Sul, Porto Alegre, 2003.

CASSAB, T. V.; ZORZETTO, N. L. Teste da fusão auditiva-revisado (AFT-R) em crianças com fissura labiopalatina. ACTA ORL: Técnicas em Otorrinolaringologia, São Paulo, v. 24, n. 4, p. 272-276, s.m. 2006.

CASSELBRANT, M. L. et al. Experimental paralysis of the tensor veli palatini muscle. Acta Oto-laryngologica, Stockholm, v. 106, n. 3-4, p.178-185, set./out. 1988.

CIELO, C. A.; CASARIN, M. T. Sons fricativos surdos. Revista CEFAC, São Paulo, v. 10, n. 3, p. 352-358, jul./set. 2008. Doi: 10.1590/S1516-18462008000300010

COSTA-FERREIRA, M. I. D. Reabilitação fonoaudiologia: do processamento auditivo à linguagem. In: CARDOSO, M. C. Fonoaudiologia na infância: avaliação e terapia. Rio de Janeiro: Revinter, 2015b. p. 73-84.

COSTA-FERREIRA, M. I. D. Reflexões sobre a avaliação de processamento auditivo na infância. In: CARDOSO, M. C. Fonoaudiologia na infância: avaliação e terapia. Rio de Janeiro: Revinter, 2015a. p. 61-72.

HOCEVAR-BOLTEZAR, I.; JARC, S.; KOZELJ, V. Ear, nose and voice problems in children with orofacial clefts. The Journal of Laryingology and Otology, Cambridge, v. 120, n. 4, p. 276-281, Apr. 2006. Doi: 10.1017/ S0022215106000454

JOHNS, D. F.; ROHRICH, R. J.; AWADA, M. Velopharyngeal incompetence: a guide for clinical evaluation. Plastic and Reconstructive Surgery, Baltimore, v. 112 , n. 7, p. 1890-1897, Dec. 2003. Doi: 10.1097/01. PRS.0000091245.32905.D5

LEMOS, I. C. C. et al. Teste Dicótico de Dígitos (etapa de escuta direcionada) em crianças com fissura labiopalatina. Revista Brasileira de Otorrinolaringologia, São Paulo, v. 74, n. 5, p. 662-667, set./out. 2008. Doi: 10.1590/ S0034-72992008000500005

LLOYD, L. L.; KAPLAN, H. Audiometric interpretation: a manual of basic audiometry. University Park Press: Baltimore, 1978.
NEVES, A. C. C.; MONTEIRO, A. M.; GIWA, N. G. H. Prevalência das fissuras labiopalatinas na Associação de Fissurados Labiopalatinos de São José dos Campos. Revista de Biociências, Taubaté, v. 8, n. 2, p. 64-74, jul./dez. 2002.

NORTHERN, J. L.; DOWNS, M. P. Audição na infância. 5 ed. Rio de Janeiro: Guanabara Koogan, 2005.

PAMPLONA, M. C. et al. Linguistic development in cleft palate patients with and without compensatory articulation disorder. International Journal of Pediatric Otorhinolaryngology, Limerick, v. 54, n. 2-3, p. 81-91, Aug. 2000. Doi: $10.1016 /$ S0165-5876(00)00332-3

PHILLIPS, S. L. et al. Frequency and temporal resolution in elderly listeners with good and poor word recognition. Journal of Speech, Language and Hearing Research, Rockville, v. 43, n. 1, p. 217-228, feb. 2000. Doi: 10.1044/jslhr.4301.217

PROCTOR, B. Anatomy of the Eustachian tube. Archives of Otolaryngology, Chicago, v. 97, n. 1, p. 2-8, jan. 1973. Doi: 10.1001/archotol.1973.00780010006002.

RUSSO, I. C.; SANTOS, T. M. M. Prática da audiologia clínica. 7. ed. São Paulo: Cortez, 2005.

SANCHEZ, M. L. et al. Avaliação do processamento auditivo em adultos. Acta Awho, São Paulo, v. 21, p. 41-48, jan./ mar. 2003.

SANTOS, F. R.; PIAZENTIN-PENNA S. H. A.; BRANDÃO, G. R. Avaliação audiológica pré-cirurgia otológica de indivíduos com fissura labiopalatina operada. Revista CEFAC, São Paulo, v. 13, n. 2, p. 271-280, mar./abr. 2011. Doi: $10.1590 /$ S1516-18462010005000028

SHINN, J. B. Temporal processing and. temporal patterning tests. In: MUSIEK, F. E.; CHERMAK, G. D. Handbook of (central) auditory processing disorder: auditory neuroscience and diagnosis. San Diego: Plural Publishing; 2007. p. 231-256.

SHINN, J. B. Temporal processing: the basics. The Hearing Journal, Baltimore, v. 56, n. 7, p. 52, jul. 2003. Doi: 10.1097/01.HJ.0000292557.52409.67

SILVA, D. P. et al. Aspectos patofisiológicos do esfíncter velofaríngeo nas fissuras palatinas. International Archives of Otorhinolaryngology, São Paulo, v. 12, n. 3, p. 426-435, jul./ set. 2008 .

SYPCZUK, G. K. Sinais e sintomas da desordem do processamento auditivo. Disponível em: $<$ http://www. neuropediatria.org.br/index.php?option=com_content\&view=article\&id=97: sinais-e-sintomas-da-desordem-doprocessamentoauditivo\&catid=59:transtorno-de-aprendizagem-escolar\&Itemid =147> . Acesso em: 15 out. 2011 . 
TEWFIK, T. L. Cleft lip and palate and mouth and pharynx deformities. Disponível em: $<$ http://emedicine. medscape.com/article/837347-overview $>$. Acesso em: 15 out. 2011.

THE CANADIAN INTERORGANIZATIONA L STEERING GROUP FOR SPEECH-LANGUAGE PATHOLOGY AND AUDIOLOGY (CISGSLPA). The Canadian guidelines on auditory processing disorders in children and adults: assessment and intervention. 2012. Disponível em: $<$ http://www.saslpa.ca/Canadian_Guidelines_on_Auditory_Processing_Disorder_in_Children_ English.pdf >. Acesso em: 15 ago. 2013.

TRINDADE, I. E. K.; TRINDADE JUNIOR, A. S. Avaliação funcional da inadequação velofaríngea. In: CARREIRÃO, S.; LESSA, S.; ZANINI, A. S. (Ed). Tratamento das fissuras labiopalatinas. 2. ed. Rio de Janeiro: Revinter, 1996. p. 223-225.
VARGAS, G. C. et al. Avaliação simplificada e comportamental do processamento auditivo em escolares: estabelecendo relações. Revista CEFAC, São Paulo, v. 16, n. 4, p. 1069-1077, jul./ago. 2014. Doi: 10.1590/1982021620142413

ZAMBONATO, T. C. F. et al. Perfil de usuários de AASI com fissura labiopalatina. Brazilian Journal of Otorhinolaryngology, São Paulo, v. 75, n. 6, p. 888-892, nov./dez. 2011.

ZORZETTO, N. L. Anatomia da orelha. In: COSTA, S. S.; CRUZ, O. L. M.; OLIVEIRA, J. A. Otorrinolaringologia: princípios e prática. Porto Alegre: Artmed, 2006. p. 23-60. 